\title{
Particle size and convergent electron diffraction patterns of triangular prismatic gold nanoparticles
}

\author{
C. Fernando-Marquez ${ }^{a}$, G. Mondragón-Galicia ${ }^{b}$, L. Bazan-Diaz ${ }^{c}$ and J. Reyes-Gasga ${ }^{a, *}$ \\ ${ }^{a}$ Instituto de Física, Universidad Nacional Autónoma de México, \\ Circuito de la Investigación Científica s/n, Cd. Universitaria. Coyoacán 04510, Ciudad de México, México. \\ *Tel. (5255) 56225083 \\ e-mails: marquezfer1560@gmail.com,*jreyes@fisica.unam.mx \\ ${ }^{b}$ Instituto de Investigaciones Nucleares, Carretera México-Toluca S/N, \\ La Marquesa, Ocoyacac, Estado. de México 52750, México. \\ e-mail: gilberto.mondragon@inin.gob.mx \\ ${ }^{c}$ Instituto de Investigaciones en Materiales, Universidad Nacional Autónoma de México, \\ Circuito Exterior s/n, Cd. Universitaria, Coyoacán 04510, Ciudad de México, México. \\ e-mail: bazanlulu@materiales.unam.mx
}

Received 9 February 2021; accepted 11 March 2021

Convergent beam diffraction (CBED) patterns of nanoparticles are possible. CBED of triangular prismatic shaped gold (Au) nanoparticle with focus on diffraction pattern symmetry and forbidden reflections observed along [111] and [112] zone axes are reported in this work. It is well known that the CBED patterns of nanoparticles of $30 \mathrm{~nm}$ or less in size only show bright kinematical discs. The dynamic contrast with Kikuchi and sharp HOLZ lines within the bright discs, as observed in CBED of volumetric materials, is well observed in particles larger of $500 \mathrm{~nm}$ in size. In addition, it is shown that the 1/3[422] and 1/2[311] weak forbidden reflections observed in the [111] and [112] electron diffraction patterns of these particles do not modify the symmetry of the CBED patterns, but they disappear as the size of the particle increases. The symmetry of the CBED patterns are always observed in concordance with the space group Fm3m (No. 225) of the Au unit cell. The possible explanations for observing forbidden reflections are the incomplete $\mathrm{ABC}$ stacking due to surface termination and the stacking faults in the fcc structure.

Keywords: Gold nanoparticles; crystal morphology; characterization; transmission electron microscopy; converging beam electron diffraction; electron diffraction.

PACS: 42.25.Fx; 61.00.00; 61.14.-x; 61.46.-w; 61.72.Nn; 68.37.Lp; 68.55.Jk; 81.00.00.

DOI: https://doi.org/10.31349/RevMexFis.67.041005

\section{Introduction}

Faced centered cubic (fcc) nanoparticles have been extensively studied for almost a century due to the scientific interest in their physicochemical properties which play an important role in optics, catalysis and electronics [1-6]. Since then, many studies have been carried out to understand their stability, growth processes, and crystallographic structure [35]. There is now an extensive work at nanometric level even on smaller particles and clusters [7]. Thus, during synthesis, particles with different shapes have been obtained, such as decahedrons, icosahedra, spheres, square, and triangular, and with different sizes. It is worth mentioning that gold $(\mathrm{Au})$ has a fcc unit cell with parameters of $0.4078 \mathrm{~nm}$ and space group Fm3m (No. 225) (PDF card 04-784).

Recent advances in transmission electron microscopy (TEM) allow obtaining electron diffraction patterns of nanoparticles as small as $5 \mathrm{~nm}$ in size, allowing to study their individual structure [8-10]. In many cases, the electron diffraction patterns of nanoparticles show "forbidden" reflections when they are observed by TEM $[11,12]$. In fcc structures, the forbidden reflections are observed along the $1 / 3$ [422] direction in the [111] zone axis diffraction pattern and along the $1 / 2$ [311] direction in the [112] zone axis diffraction pattern [11-13]. In other cases, the diffraction patterns of nanoparticles show changes in their symmetry elements [9].

New progresses have been also extensively done in convergent beam electron diffraction (CBED) for nano-sized materials. Normal CBED patterns of volumetric materials show dynamic contrast within the bright discs, as well as diffuse Kikuchi bands and sharp HOLZ lines, but the CBED patterns of nanoparticles only show bright kinematical discs [8]. In addition to this, Tehuacanero-Cuapa et al. [14], trying to obtain CBED patterns in Au decahedral nanoparticles of less than $30 \mathrm{~nm}$ in size, found that the electron beam perforated the Au nanoparticles, thus concluding that the CBED analysis should be performed with particles larger than 30-nm-in-size to avoid the perforation $[14,15]$.

The main purpose of this work is to show the CBED patterns with diffuse Kikuchi bands and sharp HOLZ lines in the bright reflection discs of triangular prismatic Au particles larger than $500 \mathrm{~nm}$, with special attention to the behavior of the $1 / 3$ [422] and $1 / 2$ [311] forbidden reflections observed in the nano-area electron diffraction (NAED) patterns along the [111] and [112] zone axis. 


\section{On the forbidden reflections}

Forbidden reflections in the fcc structures have been observed in the [111] and [112] diffraction patterns of nanoparticles [13,16-18], and in thin films [19-27]. The source of these reflections was described in terms of twin diffraction by Pashley and Stowell [16], and Davey and Deiter [17] suggested they can be indexed as an hep phase. Later, Morriss et al. [20] explained them in terms of an odd number of layers A, $\mathrm{B}$ or $\mathrm{C}$ in the stack along the direction [111], and Cherns [11] stated they can be produced by an additional atomic step in thin Au films. By computer simulation, Castaño et al. [18] indicated that their intensity is modified by the combination of size and roughness of the particle's surface.

In the case of nanostructures, Reyes Gasga et al. [12] indicated that the point or "spherical" reflections in the reciprocal space becomes "rod-like" along the normal direction to the thinnest parameter (the [111] direction in the triangular particle) due to the shape effect. The fact that reflections elongate normal to the thinnest parameter of the sample is well known [28]. Then, using a $2 \times 2$ fcc supercell in reciprocal space, Reyes-Gasga et al. [12] showed that the ZOLZ reflections for the [111] and [112] zone axes together with the "top" and "bottom" FOLZ reflections points up along the forbidden reflections. Thus, they suggest that the origin of the "forbidden" reflections could lie in the rod-like reflections when intercepting the Ewald sphere. Recently, MendozaRamírez et al [29], based on the ideas of Davey and Deiter [17] and Morriss et al. [20], suggested once again that the forbidden reflections are produced by stacking faults parallel to the surface in the triangular prismatic particles, producing the existence of two lattices in the particles. One lattice is the $\mathrm{Au}$ fcc crystal. The second lattice is a hexagonal prism along the [111] direction with $a=0.287 \mathrm{~nm}$ and $c=0.735 \mathrm{~nm}$. The space distribution of the forbidden reflections in reciprocal space following the hexagonal cell referenced to the fcc Au structure is shown in Fig. 1. Planes 111, with symmetry 6 $\mathrm{mm}$, and planes 112 , with symmetry 2 , are also presented in
Fig. 1a). Therefore, the hexagonal prism is oriented in such a way that, in the hexagonal and cubic notation, [0001]//[111] and $[10-10] / /[422]$. Figure $1 b)$ shows the projection of the reciprocal space in the [100] direction, which corresponds to Fig. 4 of Ref. [29], and Fig. 1c) shows the projection in the [111] direction. The corresponding representation in real space of Fig. 1 explains quite well the contrast observed in Fig. 5 of Ref. [12].

Following the cubic notation ( $h k l)$, the analysis of Fig. 1 indicates that the 1/3 [422] reflections are at the vertices of the hexagonal prism on the (111) plane aligned with the [111] reflections. Reflections in planes (112), located at 1/2[311], are slightly above and below the caps of the hexagonal prism, but also aligned with reflections [111].

\section{Experimental procedure}

Particles with different shapes and sizes (from around $1 \mu \mathrm{m}$ to few nanometers) were obtained by wet chemical synthesis after modifying the polyol method, as described in detail elsewhere [30,31]. Initially, the solution of ethylene glycol (EG) with $14 \mathrm{ml}$ of polyvinylpyrrolidone (PVP, Mw = $55,000)(0.7 \mathrm{M}$, in EG) was placed in a $40 \mathrm{ml}$ vial in an oil bath previously heated to $180^{\circ}$ for 30 minutes under magnetic stirring.

The temperature was then increased to $200^{\circ}$ and $1 \mathrm{ml}$ HAuCl4 solution $(0.25 \mathrm{mM}$, in EG) was immediately added dropwise to the PVP solution with vigorous magnetic stirring. The solution was stirred for 10 minutes until the solution turned into an orange-brown color, cooled to room temperature and centrifugated at $4000 \mathrm{rpm}$ with deionized water. The gold nanoparticles were re-dispersed in a mixture of ethanol/water (1:1).

For TEM observation, the solution was dissolved in ethanol and sonicated for $30 \mathrm{~s}$. A drop of the solution was deposited on a 200 mesh copper grid previously covered by plastic collodion and carbon films. TEM microscopes JEM 2010F and JEM 2020HT (Jeol, Japan), with $0.19 \mathrm{~nm}$ point- a)

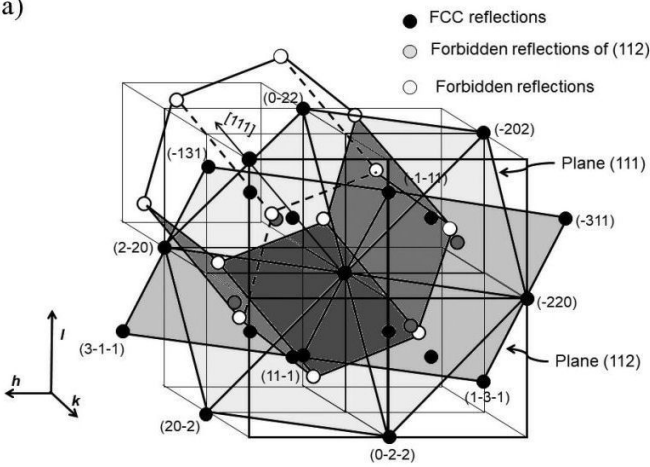

b)

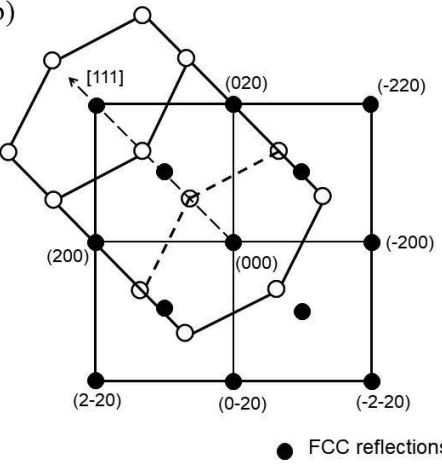

c)

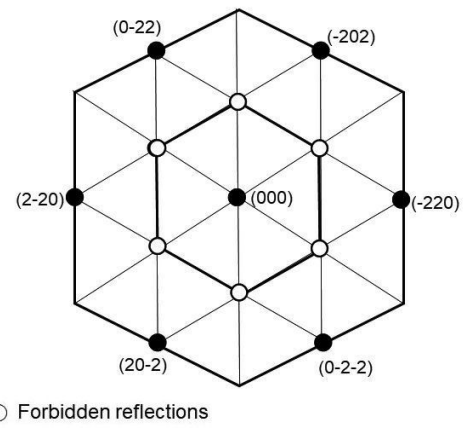

FIGURE 1. 3D representation relative to the Au fcc structure of the spatial distribution in reciprocal space of the hexagonal cell generated by forbidden reflections. The planes (111), with symmetry $6 \mathrm{~mm}$, and (112), with symmetry 2, are shown. b) Projection in the [100] direction, c) Projection in the [111] direction. 

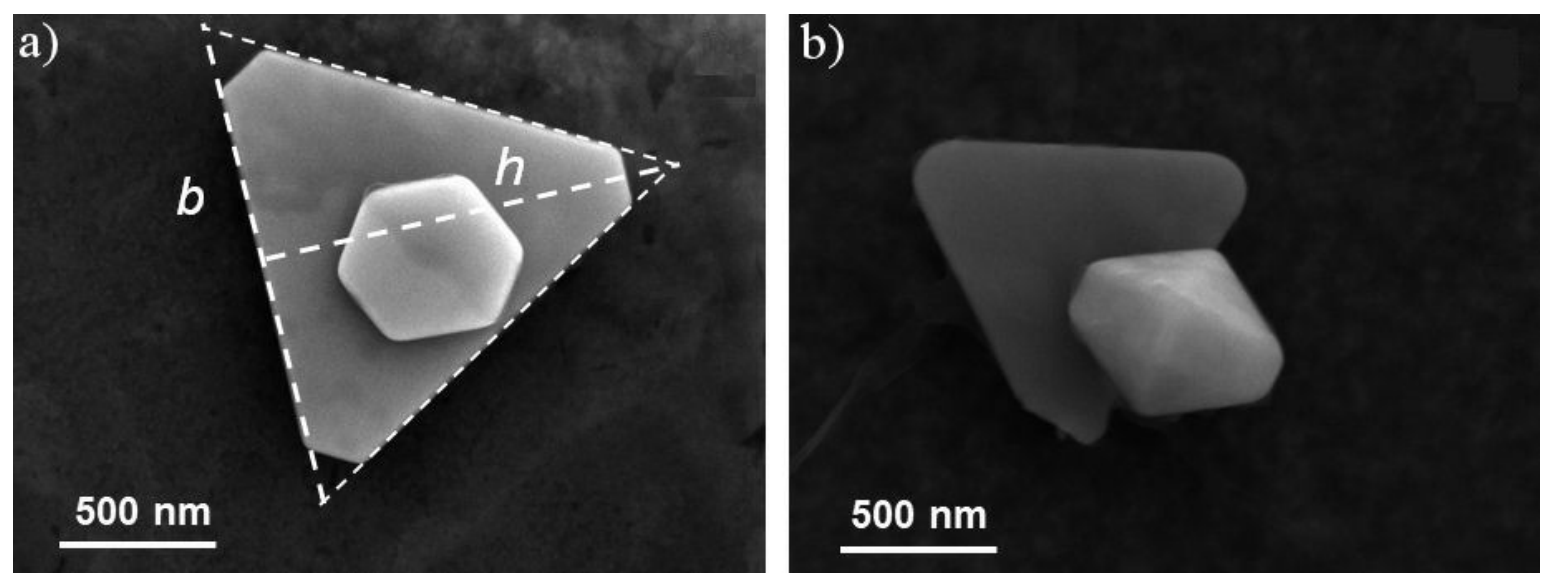

FIGURE 2. Secondary electron SEM images of two triangular prismatic Au particles. a) Particle with $b=1.5 \mu \mathrm{m}$ and $h=1.3 \mu \mathrm{m}$, with truncated vertices. b) Particle with $b=1.2 \mu \mathrm{m}$ and $h=1.1 \mu \mathrm{m}$ particle with rounded vertices. Particles of different shapes are observed on top of the triangular particles.
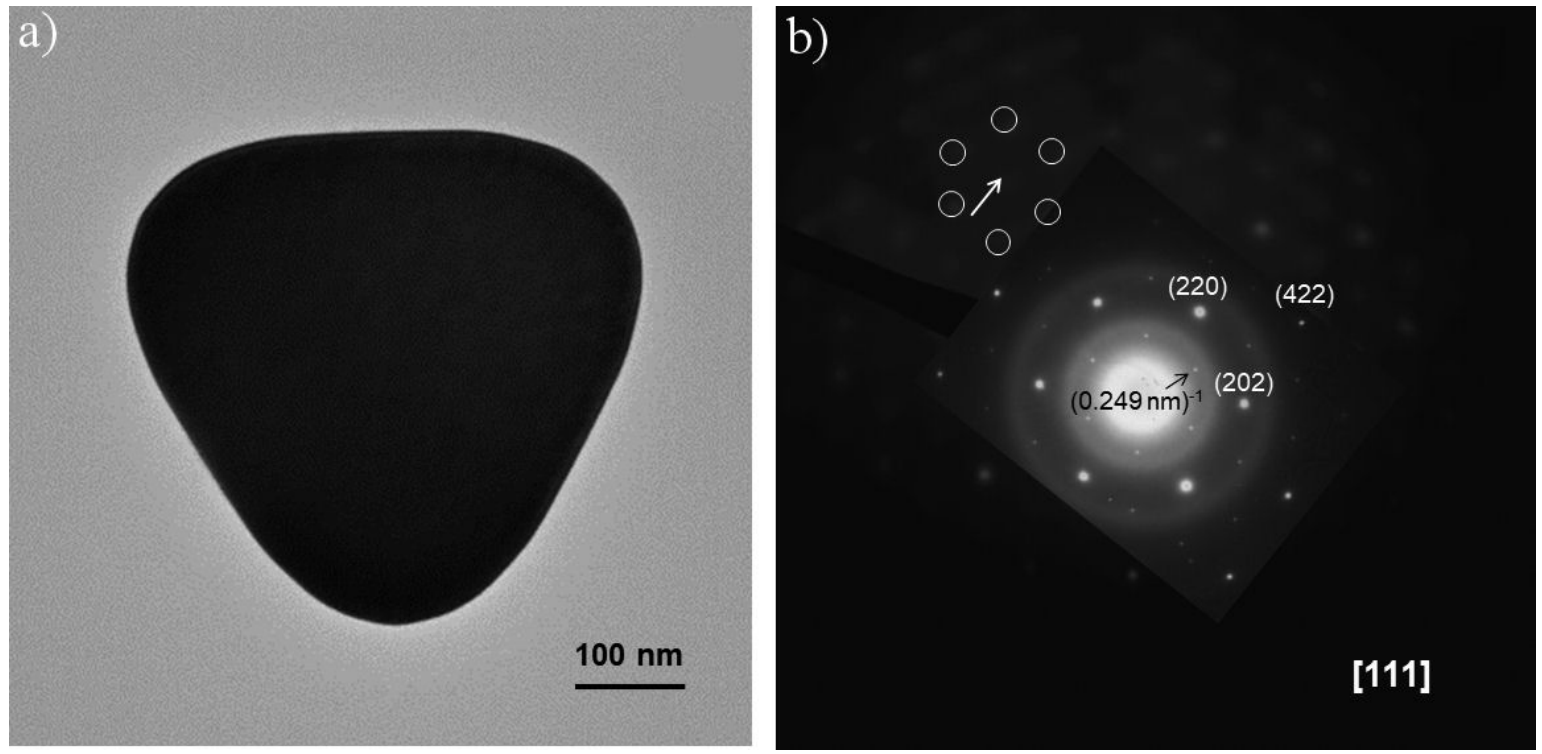

FIGURE 3. a) TEM bright field image of a triangular prismatic particle with $b=690 \mathrm{~nm}$ and $h=590 \mathrm{~nm}$ with rounded vertices. b) NAED pattern in the [111] direction. Note the $(0.249 \mathrm{~nm})^{-1}$ weak reflections. The insert in (B) shows the [111] diffraction pattern of a 30-nm-in-size triangular particle.

to-point resolution and with a $\pm 15^{\circ} \mathrm{C}$ double-tilt specimen holder, were used operated at $200 \mathrm{kV}$. A CMOS digital camera (Gatan, USA) was used for the digital acquisition of images NAED and CBED patterns. A SEM JSM7800F microscope (Jeol, Japan) operated at $15 \mathrm{kV}$ was used for the scanning electron microscopy (SEM) observations. The parameters for the CBED patterns were: accelerating voltage $200 \mathrm{kV}$, beam diameter of $2.0 \mathrm{~nm}$, emission current of $150 \mu \mathrm{A}$, convergence half angle $\alpha$ of $3 \mathrm{mrad}$, and camera length $L$ from 10 to $20 \mathrm{~cm}$.

\section{Results}

The obtained triangular prismatic Au particle is not quite a straight triangular prism since, although its base is triangular, the surface of the rectangular faces is slightly curved, but it is a good approximation. Figure 2 shows two SEM images of these particles. In TEM, the diffraction patterns indicated that the [111] zone axis is perpendicular to the triangular surface of the particle. Figure 2 a) shows a particle with rounded corners, and where the parameters of length $b$ and height $h$ have been defined. These two parameters are used throughout this work to describe the sizes of the particles. In the case particles with truncated or rounded vertices, as shown in Fig. 2, lines parallel to the edges were drawn to obtain the corresponding value of $b$ and $h$. Then, for the particle in Fig. $2 \mathrm{a}), b=1.5 \mu \mathrm{m}$ and $h=1.3 \mu \mathrm{m}$, while for the particle in Fig. 2b), $b=1.2 \mu \mathrm{m}$ and $h=1.1 \mu \mathrm{m}$. 

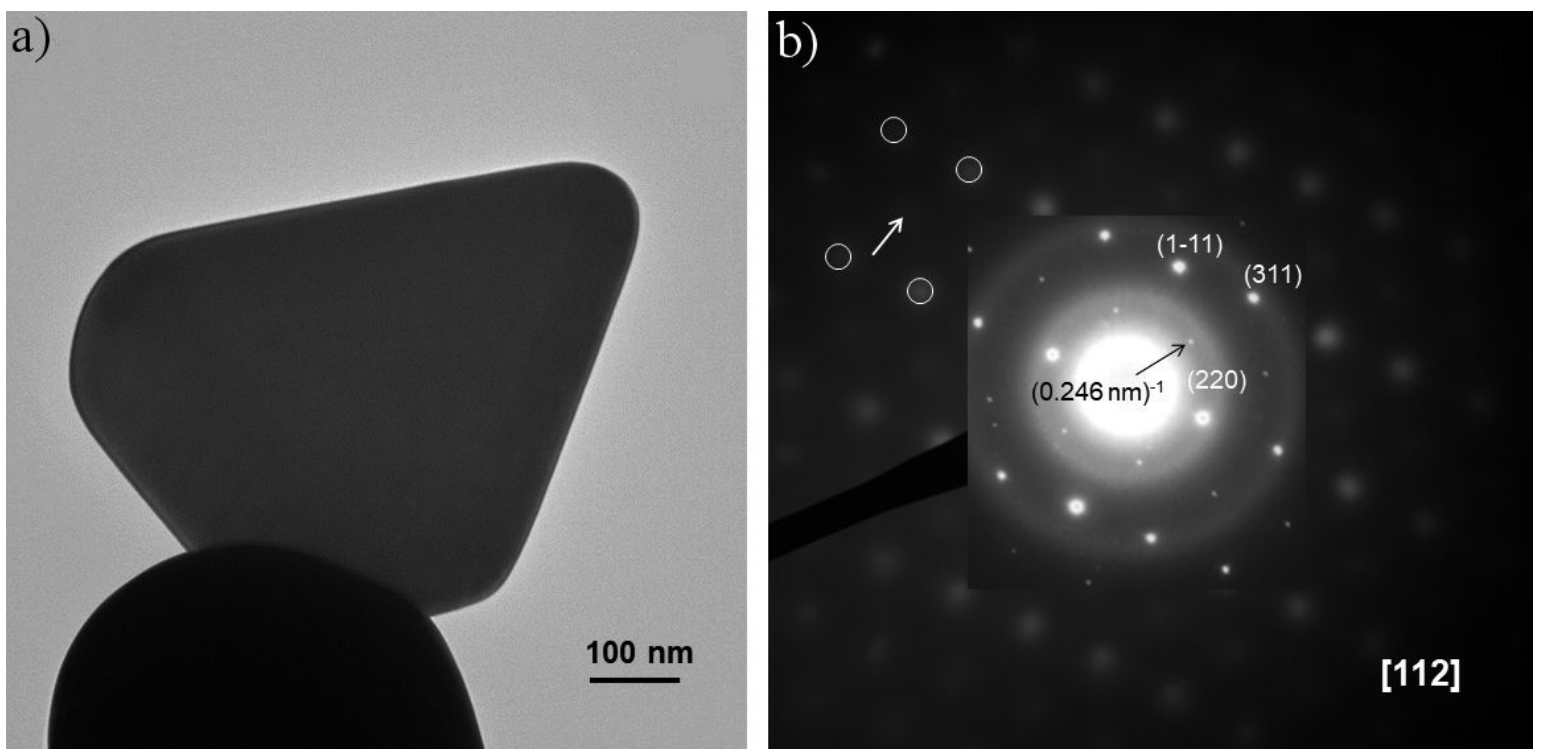

FIGURE 4. a) TEM bright field image of a triangular prismatic Au particle with $b=880 \mathrm{~nm}$ and $h=770 \mathrm{~nm}$ with rounded vertices. b) NAED pattern in the [112] direction where the $(0.246 \mathrm{~nm})^{-1}$ weak forbidden reflections are observed (indicated by the arrow). The insert in (B) shows the [112] diffraction pattern of a $30 \mathrm{~nm}$-size triangular particle.
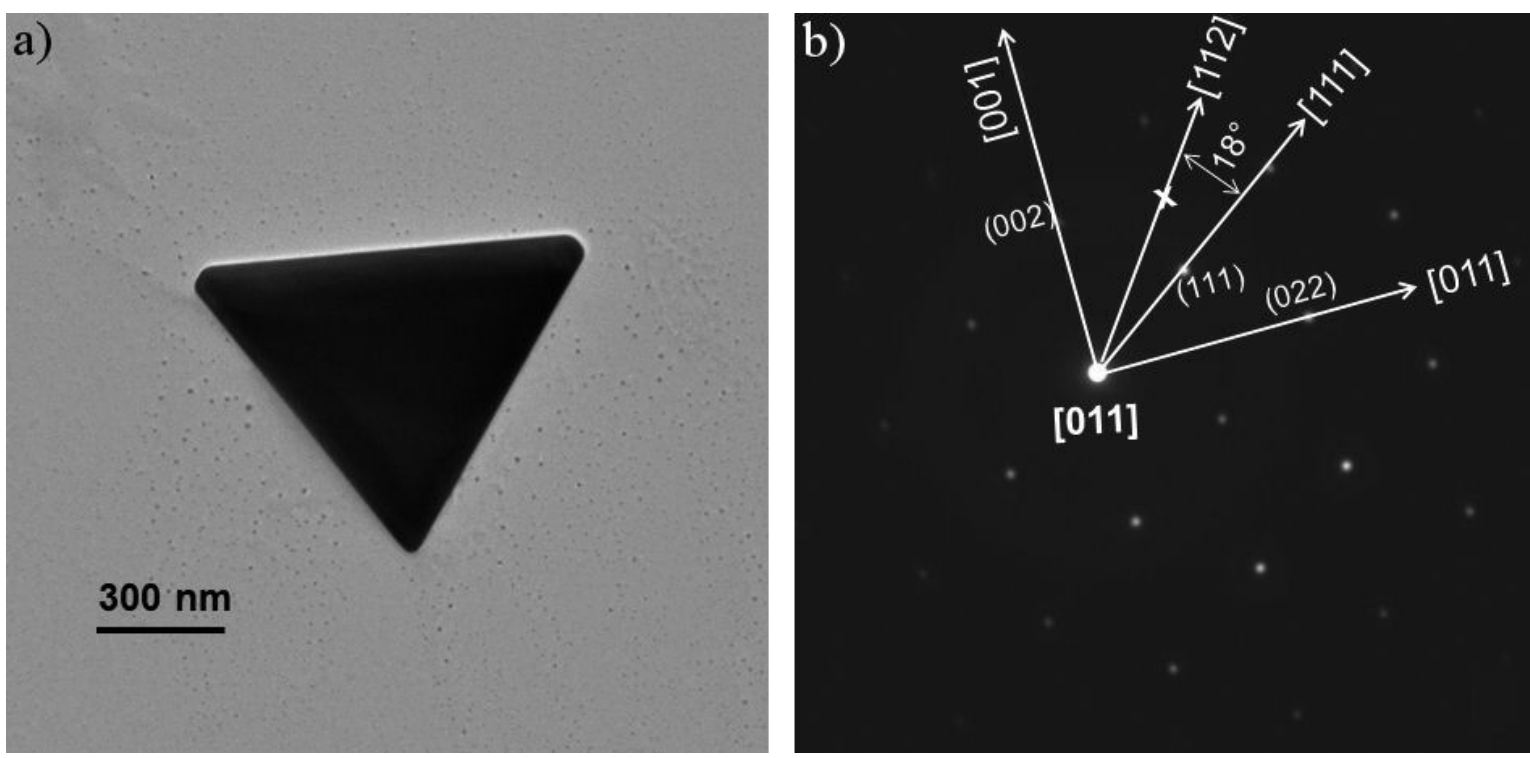

FIGURE 5. a) TEM bright field image of a triangular prismatic Au particle with $b=1.0 \mu \mathrm{m}$ and $h=825$ nm with slightly rounded vertices. b) NAED pattern in the [011] direction. No forbidden reflections are observed in this direction. The angle between [111] and [112] directions is indicated in (B).

\subsection{Electron diffraction}

Figure 3a) shows the bright field TEM image of a triangular prismatic Au particle with $b=690 \mathrm{~nm}$ and $h=590$ $\mathrm{nm}$ with rounded vertices together with the [111] electron diffraction pattern (Fig. 3b). In the electron diffraction pattern, the $(0.249 \mathrm{~nm})^{-1}$ weak reflections corresponding to the 1/3[422] forbidden reflections can be seen. These forbidden reflections are weak in intensity due to the size of the particle: larger the particle, weaker is the forbidden reflection. The insert in Fig. 3b) shows the [111] diffraction pattern of a 30-nm-in-size triangular particle where the forbidden reflections are more defined. Including the forbidden reflections, the hexagonal lattice presents the $6 \mathrm{~mm}$ point group.

In the case of the [112] zone axis, Fig. 4a) shows the bright field TEM image of a triangular prismatic Au particle with $b=880 \mathrm{~nm}$ and $h=770$. Figure $4 \mathrm{~b}$ ) shows the [112] electron diffraction pattern and the $(0.246 \mathrm{~nm})^{-1}$ reflections, which correspond to the 1/2[311] weak forbidden reflections. The insert in Fig. 4b) shows the [112] diffraction pattern of a $30 \mathrm{~nm}$-size triangular particle where the forbidden reflections are observed more defined. In this case, the forbidden reflec- 

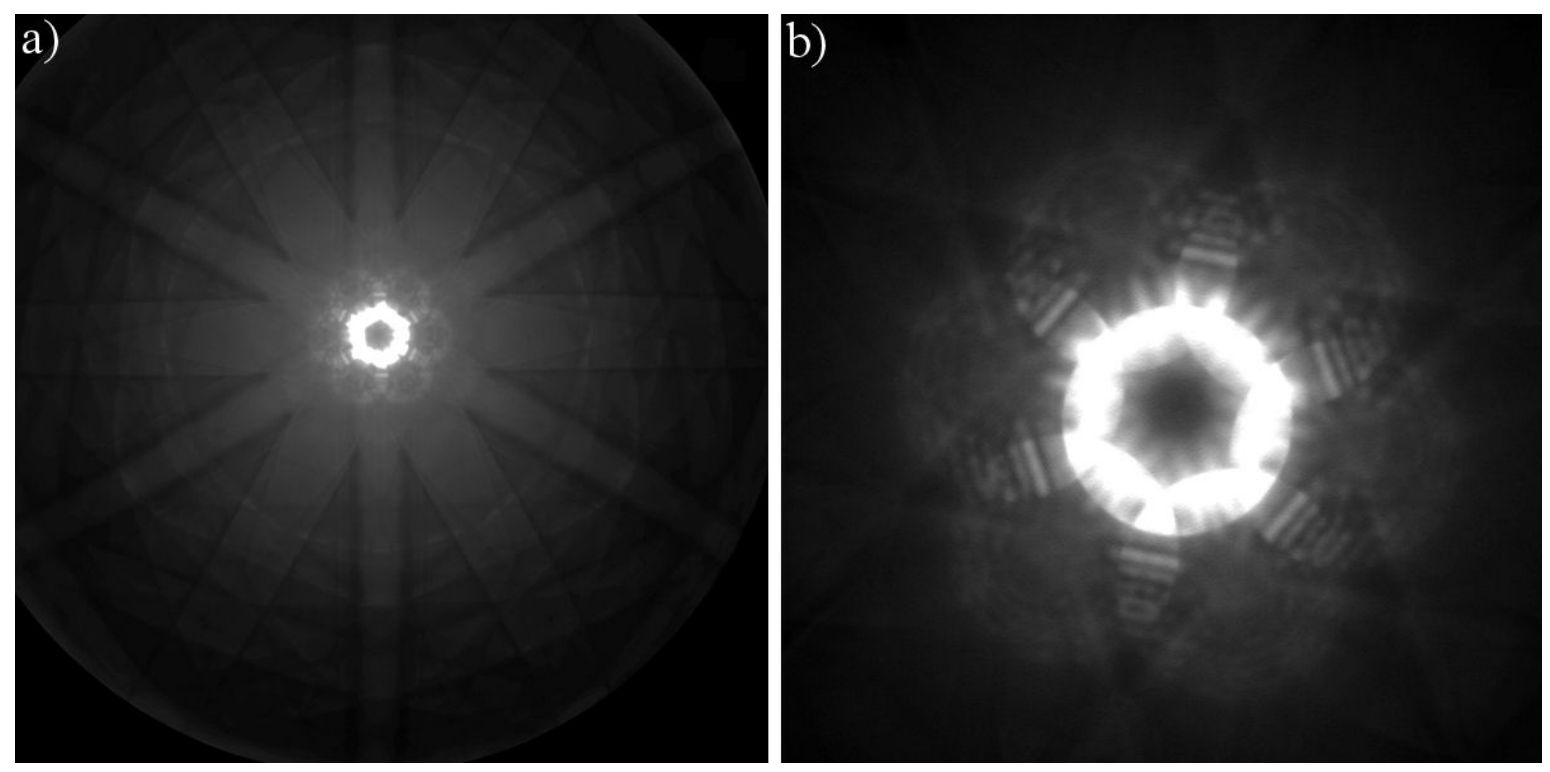

FIGURE 6. CBED patterns in the [111] direction of the triangular prismatic Au particle shown in Fig. 3. a) The Kossel pattern with the 6 mm point group; camera length $8 \mathrm{~cm}$, spot size $25 \mathrm{~nm}$, semi-convergence angle 3 mrad. b) The (000) disc and surrounding \{220\} discs with the $6 \mathrm{~mm}$ point group (slightly outside the zone axis); camera length $20 \mathrm{~cm}$, spot size $25 \mathrm{~nm}$, semi-convergence angle 3 mrad. The rings of excess HOLZ lines in (A) show the $3 \mathrm{~m}$ point group.

tions produce a centered rectangular Bravais lattice with a point group 2.

In the triangular particles, the forbidden reflections were only observed in the [111] and [112] diffraction patterns. Figure 5 shows the TEM bright field image and the corresponding [011] electron diffraction of a triangular prismatic $\mathrm{Au}$ particle with $b=1.0 \mu \mathrm{m}$ and $h=825 \mathrm{~nm}$. Forbidden reflections are not observed in this zone axis and, therefore, the centered rectangular lattice shows the point group 2. In Fig. 5b) the [111] and [112] directions and the angle between them, which is approximately $18^{\circ}$, are shown.

\subsection{CBED patterns}

The dynamic contrast with diffuse Kikuchi bands and sharp HOLZ lines within the bright discs, are observed in particles equal or larger than $500 \mathrm{~nm}$. Figure 6 shows the [111] CBED pattern of the triangular prismatic Au particle shown in Fig. 3. Figure 6a) shows the CBED pattern in the borderline of a Kossel pattern and, therefore, it presents the Kikuchi and HOLZ lines with the $6 \mathrm{~mm}$ point group distribution. The rings of excess HOLZ lines show the $3 \mathrm{~m}$ point group. Figure $6 \mathrm{~b})$ shows the (000) disc and surrounding $\{220\}$ discs with dynamic contrast forming the $6 \mathrm{~mm}$ point group (although slightly outside the zone axis). All these symmetries are in agreement with the Au symmetry along the [111] direction.

Forbidden reflections are indeed included in CBED patterns. Figure 7 shows the [112] CBED pattern of the triangular prismatic Au particle shown in Fig. 4. This pattern shows dynamic contrast within the CBED discs with the 2 point group (slightly outside the zone axis). The insert in this figure shows the [112] diffraction pattern indicating the
$1 / 2$ [311] forbidden reflections from which the discs shown in the CBED pattern come from.

Figure 8a) shows the [110] CBED pattern of the triangular prismatic Au particle shown in Fig. 5 in the borderline of a Kossel condition. This pattern shows the Kikuchi lines and HOLZ lines with the $2 \mathrm{~mm}$ point group for the whole-pattern

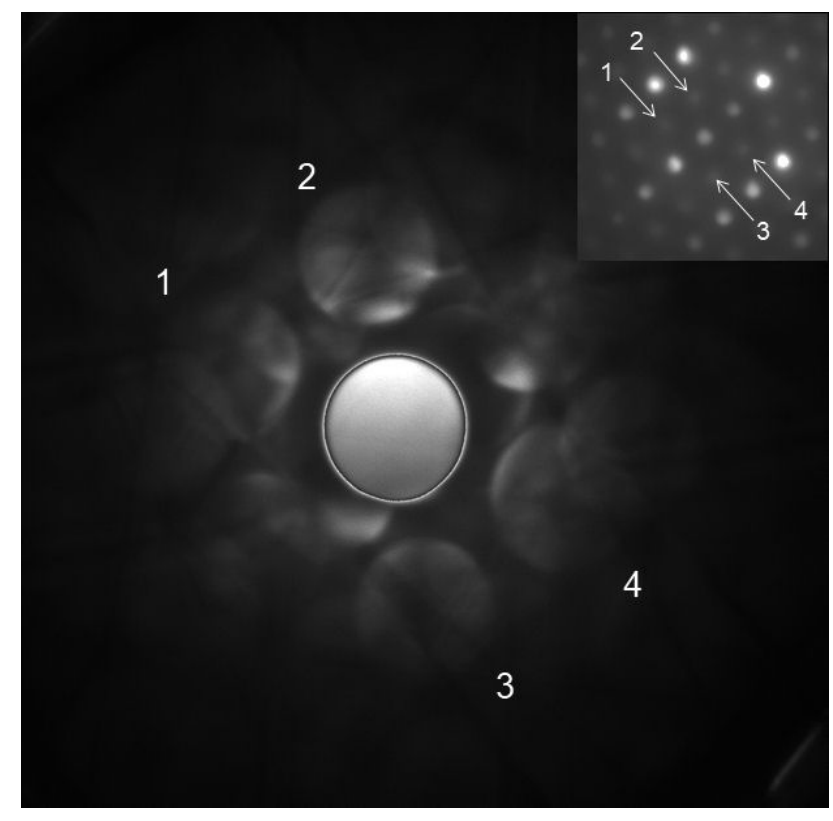

FIGURE 7. CBED pattern in the [112] direction of the triangular prismatic $\mathrm{Au}$ particle shown in Fig. 4 presenting the 2 point group (slightly outside the zone axis); camera length $10 \mathrm{~cm}$, spot size 25 $\mathrm{nm}$, semi-convergence angle $3 \mathrm{mrad}$. The insert shows the [112] diffraction pattern indicating the forbidden reflections 1 to 4 which appear as discs in the CBED. 

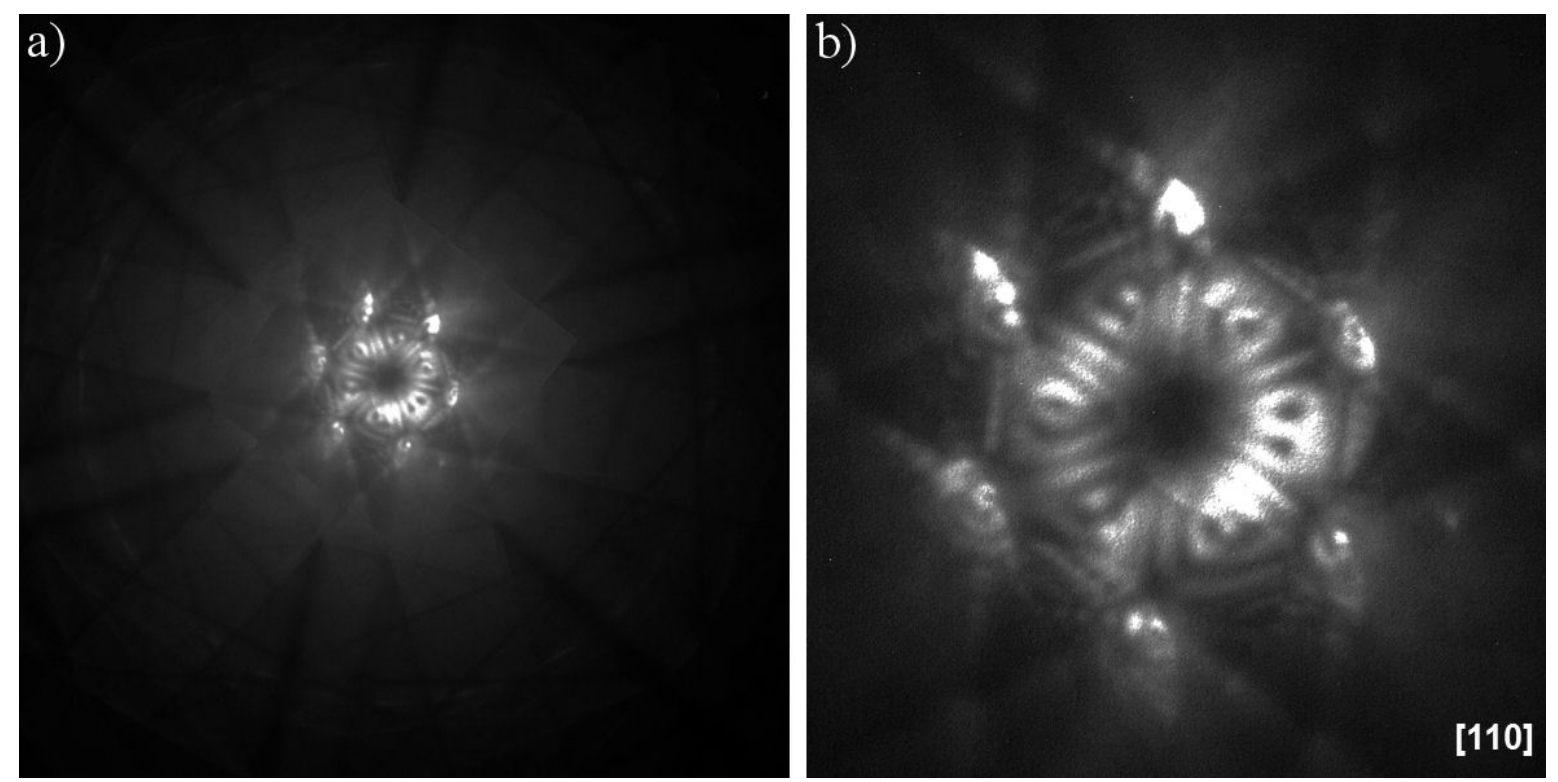

FIGURE 8. CBED pattern in the [110] direction of the triangular prismatic Au particle shown in Fig. 5. a) The Kossel pattern showing the $2 \mathrm{~mm}$ point group as the whole-pattern symmetry; camera length $10 \mathrm{~cm}$, spot size $3 \mathrm{~nm}$, semi-convergence angle $3 \mathrm{mrad}$. b) the (000) disc and surrounding by the $\{110\}$ and $\{002\}$ discs presenting the 2 point group (slightly outside the zone axis); camera length $18 \mathrm{~cm}$, spot size $3 \mathrm{~nm}$, semi-convergence angle $3 \mathrm{mrad}$.

symmetry, as expected for the fcc structures in this direction. Figure $8 b$ ) shows the (000) disc surrounding by the $\{110\}$ and $\{002\}$ discs with dynamic contrast. The CBED pattern shows the 2 point group (slightly outside the zone axis).

Therefore, the CBED observed symmetries are in concordance with the Fm3m space group of the Au fcc unit cell, and the forbidden reflections positions are compatible with these symmetries.

\section{Discussion}

Triangular prismatic particles are one of the most interesting nanoparticle shapes due to their two-dimensional growth: their velocity growth in the plane is much greater than in the perpendicular direction, the [111] direction. Thanks to the fact that they are in different sizes and thicknesses, and that an important question in the nanoparticles and in the field of electron diffraction is to know the thickness value from which dynamic dispersion is present in the CBED patterns, their study indicate the range of utility of this type of patterns in the crystallography study of defects of the nanometric structures. It is well known that depending on the thickness of the TEM samples, the discs of the CBED pattern have or do not have dynamic contrast $[28,32]$. If the thickness is less than the extinction distance $\xi_{g}$, kinematic conditions will be obtained and the discs will be bright without contrast inside. If it is greater than $\xi_{g}$, the discs will present dynamic contrast. The $\xi_{g}$ values of $\mathrm{Au}$ for (111) is $18.3 \mathrm{~nm}$, for (200) is 20.2 $\mathrm{nm}$ and for (220) it is 27.8 .

Let $t$ be the thickness of the triangular prismatic particles. In this way, the lateral faces of the triangular prism can be square or rectangular, depending on the value of $t$. If $t=b$, the faces are square, if $t \neq b$, the faces are rectangular. For TEM observation, samples should have an average thickness of $10 \mathrm{~nm}$, which clearly indicate that $t<b$, so their faces are rectangular with length parallel to $b$ and width parallel to $t$.

Some approximations of the $t / b$ ratio can be obtained from the TEM images of the triangular prismatic particles reported in literature [12,29] where the thickness of the triangular particles were obtained by tilting. These TEM images indicate that the ratio $t / b$ is between 0.1 and 0.3 [29], being the value of 0.1 suitable for the thickness to obtain the high resolution TEM (HRTEM) images [12]. Taking an average value of 0.2 for the ratio $t / b$, an approximation for the thickness of the triangular prismatic particles shown in this work is obtained. By performing this analysis to the particles shown in reference [29], the approximation for $t$ with an error of $14 \%$ on average is obtained. Thus, the particle in Fig. 2a) should have a $t$ of $307 \mathrm{~nm}$ and for the one in Fig. 2b); $t$ is of $247 \mathrm{~nm}$, approximately. For the particle shown in Fig. 3a), $t=138 \mathrm{~nm}$; for the particle shown in Fig. $4 \mathrm{a}, t=176 \mathrm{~nm}$; and for the particle shown in Fig. 5a), $t=205 \mathrm{~nm}$.

Therefore, results indicate that when as the particle size is above $500 \mathrm{~nm}$ in length and $100 \mathrm{~nm}$ in thickness:

- they are adequate to produce dynamic and inelastic scattering.

- the CBED patterns show dynamic contrast with Kikuchi bands and sharp HOLZ lines.

- the forbidden reflections fade away.

The fact that these forbidden reflections disappear as the particle grows implies a possible phase transition from the 
hexagonal lattice arrangement that the forbidden reflections present towards the macroscopic fcc lattice of Au as the particle grows. For the nucleation of the triangular prismatic particles there should be a seed with hexagonal symmetry; this hexagonal structure could be related with the Auhcp phase [33], but with the lattice parameters obtained from Fig. 1 for the Auhcp are: $a=0.296 \mathrm{~nm}$ and $c=0.484 \mathrm{~nm}$. The growth of the particle is anisotropic occurring mainly in two dimensions, adding few planes in the [111] direction. As the triangular prismatic particle reach a micron size approximately, it becomes less stable, and it changes to the Aufcc [33]. Wang et al. [34] have indicated, after first-principles calculations, that the phase transformation Auhcp to Aufcc is favored by the appearance of stacking faults. The stacked planes are found in the [111] Aufcc ([0001] Auhcp) direction, Thus, the mechanism of growth through stacking faults could explain the triangular prismatic particles. In addition, the triangular shape of these particles also suggests the existence of an odd number of twin planes parallel to the surface.

\section{Conclusions}

CBED patterns of triangular prismatic Au nanoparticles with dynamic contrast of Kikuchi and HOLZ sharp lines are ob- served when they are larger than $500 \mathrm{~nm}$ in size. The symmetries observed in these CBED patterns are compatible with the Fm3m symmetry of the Au fcc unit cell. The weak forbidden reflections $1 / 3$ [422] and $1 / 2$ [311] observed in the nanodiffraction patterns along the [111] and [112] zone axes are also observed in the CBED patterns, implying that they compatible with the Au fcc structure.

\section{Declaration of competing interest}

The authors declare that they have no known competing financial interests or personal relationships that could have appeared to influence the work reported in this paper.

\section{Acknowledgments}

The authors would like to thank Prof. Dr. M. J. Yacaman for the comments regarding the possible origin of forbidden reflections in gold nanoparticles. Also to S. TehuacaneroCuapa, S. Tehuacanero-Nuñez, R. Hernández, P. López for their technical support in the elaboration of this work. This work was carried out with the partial financial support granted by DGAPA-UNAM through the project IN101319.
1. . B.G. Bagley, A dense packing of hard spheres with five-fold symmetry, Nature 208 (1965) 674. http:dx.doi.org/ $10.1038 / 208674 a 0$.

2. S. Ino, Epitaxial growth of metals on rock salt faces cleaved in vacuum. II. Orientation and structure of gold particles formed in ultrahigh vacuum, J. Phys. Soc. Japan 21 (1966) 346. http:dx.doi.org/10.1143/JPSJ.21.346.

3. L. D. Marks, and A. Howie, Multiply-twinned particles in silver catalysts, Nature 282 (1979) 196, https://doi .org/ $10.1038 / 282196 \mathrm{a} 0$

4. C. Y. Yang, M. Jose-Yacaman, and K. Heinemann, Crystallography of decahedral and icosahedral particles, J. Cryst. Growth 47 (1979) 283, http:dx.doi.org/10.1016/ 0022-0248(79) 90253-7.

5. D. J. Smith and L. D. Marks, Direct lattice imaging of small metal particles, Phil. Mag. A 44 (1981) 735, https : / / doi. org/10.1080/01418618108236175

6. A. Mayoral, H. Barron, R. Estrada-Salas, A. Vazquez-Duran and M. José-Yacamán, Nanoparticle stability from the nano to the meso interval, Nanoscale 2 (2010) 335, http: dx. doi. org/10.1039/B9NR00287A

7. R. B. Neder, and T. Proffen, Diffuse scattering and defect structure simulations: A cook book using the program DISCUS. (Oxford University Press, 2008). http:dx.doi.org/10. 1093/acprof:oso/9780199233694.001.0001.

8. R. V. Petrova, R. R. Vanflett, D. R. Richardson, B. Yao, and K. R. Coffey, Convergent beam electron diffraction of ordered L1o nanoparticles, Microsc. Microanal. 11 (2005) 782, http: dx.doi.org/10.1017/S1431927605506901
9. L. D. Romeu, and J. Reyes-Gasga, Interpretation of the nanoelectron-diffraction patterns along the five-fold axis of decahedral gold nanoparticles, Microsc. Microanal. 17 (2011) 279, http:dx.doi.org/10.1017/S1431927610094511

10. A. Bhattacharya, C. M. Parish, J. Henry, and Y. Katoh, High throughput crystal structure and composition mapping of crystalline nanoprecipitates in alloys by transmission Kikuchi diffraction and analytical electron microscopy, Ultramicroscopy 202 (2019) 33, https : / / doi .org/10.1016/ j.ultramic.2019.03.015

11. D. Cherns, Direct resolution of surface atomic steps by transmission electron microscopy, Phil. Mag. 30 (1974) 549, http: $\mathrm{dx}$.doi.org/10.1080/14786439808206580

12. J. Reyes-Gasga, A. Gomez-Rodriguez, X. Gao, and M. JoseYacaman, On the interpretation of the forbidden spots observed in the electron diffraction patterns of flat $\mathrm{Au}$ triangular nanoparticles, Ultramicroscopy 108 (2008) 929, http: dx.doi.org/10.1016/j.ultramic.2008.03.005

13. M. K. Singh, B. Mukherjee, and R. K. Mandal, Growth morphology and special diffraction characteristics of multifaceted gold nanoparticles, Micron 94 (2017) 46, https://doi. org/10.1016/j.micron.2016.12.002

14. S. Tehuacanero-Cuapa, R. Palomino-Merino, and J. ReyesGasga, CBED electron beam drilling and closing of holes in decahedral silver nanoparticles, Radiat. Phys. Chem. 87 (2013) 59, http:dx.doi.org/10.1016/j. radphyschem.2013.02.023 
15. S. Tehuacanero-Cuapa, J. Reyes-Gasga, E. F. Brés, R. Palomino-Merino, and R. García-García, Holes drilling in gold and silver decahedral nanoparticles by the CBED electronbeam, J. Radiat. Eff. Defects Solids 169 (2014) 838, http: dx.doi.org/10.1080/10420150.2014.958747

16. D. W. Pashley, and M. J. Stowell, Electron microscopy and diffraction of twinned structures in evaporated films of gold, Phil. Mag. 8 (1963) 1605, http:dx.doi.org/10.1080/ 14786436308207327

17. J. E. Davey, and R. H. Deiter, Structure in textured gold films, $J$. Appl. Phys. 36 (1965) 284, http: dx . doi.org/10.1063/ 1.1713891 .

18. V. Castaño, A. Gomez, and M. José-Yacamán, Microdiffraction and surface structure of small gold particles, Surf. Sci. 146 (1984) L587, http:dx.doi.org/10.1016/ 0039-6028(84)90429-1.

19. D. W. Pashley, M. J. Stowell, M. H. Jacobs, and T. J. Law, The growth and structure of gold and silver deposits formed by evaporation inside and electron microscope, Phil. Mag. 10 (1964) 127. http:dx.doi.org/10.1080/ 14786436408224212

20. R. H. Morriss, W. R. Bottoms, and R. G. Peacock, Growth and defect structure of lamellar gold microcrystals. J. Appl. Phys. 39 (1968) 3016. http: dx.doi.org/10.1063/1. 1656724

21 . R. L. Hines, Surface structures on thin gold and platinum crystals, Thin Solid Films 35 (1976) 229, http:dx.doi.org/ 10.1016/0040-6090(76) 90260-1.

22. J. C. Heyraud, and J. J. Metois, Anomalous 13422 diffraction spots from $\{111\}$ flat gold crystallites: (111) surface reconstruction and moiré fringes between the surface and the bulk, Surf. Sci. 100 (1980) 519, http:dx.doi.org/10.1016/ 0039-6028(80) 90419-7

23. Y. Tanishiro, H. Kanamori, K. Takayanagi, K. Yagi, and G. Honjo, UHV transmission electron microscopy on the reconstructed surface of (111) gold: I. General features, Surf. Sci. 111 (1981) 395, http:dx.doi.org/10.1016/ 0039-6028(81) 90397-6

24. A. I. Kirkland et al., Structural studies of trigonal lamellar particles of gold and silver, Proc. R. Soc. Lond. A 440 (1993) 589. DOI: $10.1098 /$ rspa.1993.0035
25. V. Germain, J. Li, D. Ingert, Z. L. Wang, and M. P. Pileni, Stacking faults in formation of silver nanodisks, J. Phys. Chem. B 107 (2003) 8717, http:dx .doi.org/10.1021/ jp0303826

26. B. Rodriguez-González, I. Pastoriza-Santos, and L.M. LizMarzan, Bending contours in silver nanoprisms, J. Phys. Chem. B 110 (2006) 11796, http:dx.doi.org/10. $1021 /$ jp0611951

27. A. Courty, A. I. Henry, N. Goubet, and M. P. Pileni, Large triangular single crystals formed by mild annealing of self-organized silver nanocrystals, Nat. Mater. 6 (2007) 900, https:// doi.org/10.1038/nmat2004

28. P. B. Hirsch, A. Howie, R. B. Nicholson, D. W. Pashley, and M.J. Whelan, Electron microscopy of thin crystals, (Krieger Publishing Company, New York, 1977).

29. M. C. Mendoza-Ramírez, H. G. Silva-Pereyra, and M. Avalos-Borja, Hexagonal phase into Au plate-like particles: A precession electron diffraction study, Mater. Charact. 164 (2020) 110313. http://doi.org/10.1016/j. matchar.2020.110313

30. F. Fievet, J. P. Langier, B. Blin, B. Beaudoin, and M. Figlarz, Homogeneous and heterogeneous nucleation in the polyol process for the preparation of micron and submicron size metal particles, Solid State Ionics 32 (1989) 198, http://doi. org/10.1016/0167-2738(89)90222-1

31. U. Santiago et al., A stable multiply twinned decahedral gold nanoparticle with barrel-like shape, Surf. Sci. 644 (2016) 80, http://doi.org/10.1016/j.susc.2015.09.015

32. D. B. Williams and C. B. Carter, Transmission Electron Microscopy, 2nd ed., Ch. 20 (Springer, Boston, 2009), https: //doi.org/10.1007/978-0-387-76501-3

33. X. Huang et al., Synthesis of hexagonal close-packed gold nanostructures, Nat. Commun. 2 (2011) 292 https : / / doi. org/10.1038/ncomms 1291 .

34. C. Wang et al., Generalized- stacking-fault energy and twinboundary energy of hexagonal close-packed Au: a firstprinciples calculation, Sci. Rep. 5 (2015) 10213, https: // doi.org/10.1038/srep10213. 